\title{
ON THE PRODUCT AND RATIO OF TWO GENERALIZED ORDER STATISTICS FROM THE GENERALIZED BURR TYPE-II DISTRIBUTION
}

\author{
Mohamed Maswadah \\ Department of Mathematics, Faculty of Science, Aswan University, Aswan, Egypt
}

Received 2012-11-29, Revised 2012-12-06; Accepted 2013-05-10

\begin{abstract}
In this study, the probability density functions of the product and quotient of two generalized order statistics from the generalized Burr Type-XII distribution have been derived, using the Mellin transform and its inverse. Several interesting special cases have also been obtained such as the product and quotient of extreme generalized order statistics and the product and quotient of consecutive generalized order statistics.
\end{abstract}

Keywords: Generalized Order Statistics, Mellin Transform Technique, Hyper Geometric Function, Fox HFunction, Generalized Burr Type-XII Distribution

\section{INTRODUCTION}

The Exponentiated distributions can be obtained by two common techniques: Firstly, Powering a positive real number to the Cumulative Distribution Function $(\mathrm{CDF})$, i.e., if we have $\mathrm{CDF} F(\mathrm{x})$ of any random variable $X$, then the function $\mathrm{F}(\mathrm{z})=[\mathrm{F}(\mathrm{z})]^{\theta}, \theta>0$, is called an exponentiated distribution Gupta and Kundu (1999; 2001); Mudholkar and Srivastava (1993) and Nadarajah (2005). Secondly, using the formula $F(z)=1-[1-F(z)]^{\theta}$ Nadarajah (2005).

The aim of this study is to study a distribution which generalizes the standard Burr Type-XII distribution using the second technique. Thus, the Probability Density Function (PDF) and the CDF of the exponentiated Burr Type XII distribution (EB), are given respectively as Equation 1 and 2:

$$
\begin{aligned}
& f(x)=\alpha \beta \theta x^{-\alpha-1}\left(1+x^{-\alpha}\right)^{-\beta-1} \\
& {\left[1-\left(1+x^{-\alpha}\right)^{-\beta}\right]^{\theta-1}} \\
& F(x)=1-\left[1-\left(1+x^{-\alpha}\right)^{-\beta}\right]^{\theta}
\end{aligned}
$$

$\alpha, \beta>0$ are shape parameters and $\theta$ is called the exponentiated parameter.

The Burr type-XII model has been considered by Burr (1942), as a new lifetime distribution and has been studied extensively in the literature as an appropriate and useful failure model to the applied statistics. Evans and Simon (1975) discussed the Burr distribution as a failure model and derived the Maximum Likelihood Estimators (MLE) for its parameters. Evans and Ragab (1983) discussed the Bayes estimotors for Burr distribution paramaeters based on type-II censored samples. Wu and $\mathrm{Yu}$ (2005) proposed pivotal quantities to test the shape parameter and established its confidence interval based on censored data. Soliman (2002) derived the MLE and Bayes estimates for the parameters. Xiuchun et al. (2007) derived the empirical Bayes estimator of the reliability based on progressively type-II censored samples and Wang and Shi (2010) derived the empirical Bayes based on record values. For intensive review for the Burr type-XII distribution, Rodiguez (1977).

However, in recent years, this distribution has been used in a variety of fields such as business Rodriguez and Taniguchi (1980). Economics and finance McDonald and Richards (1987). Hydrology, Mielke and Johanson (1974). Quality assurance, Burr (1976). Medicine, Wingo (1993) and Mineralogy, Cook and Johnson (1986). Lee et al. (2009) obtained the Bayes and empirical Bayes estimators for the reliability. Thus several aspects of the distribution have been covered by researchers, but a comprehensive statistical analysis on the distribution is still lacking.

The distributions of the product and the quotient of two random variables are widely used in many areas of statistical analysis. An important example of 
products of random variables is the case of investment in a number of different overseas markets. An important example for the quotient of order statistics is the stress-length model within the context of reliability. It describes the life of a component which has a random stress $\mathrm{Y}$ and is subject to random stress $X$. The component fails at the instant that stress applied to it exceeds the strength. The distribution of product and quotient have been studied by several authors, Subrahmaniam (1970); Wallgren (1980); Malik and Trudel (1982); Tang and Gupta (1984); Nadarajah (2005); Nadarajah and Gupta (2005a; 2005b); Nadarajah and Kotz (2007); Aleem (2008); Garg (2009) and Nagar and Valencia (2011). They are met in the problem of selection and ranking rules, which is described by Gulati (1970) and Malik (1970). They also can occur when the random variables have dimensions of a ratio such as cost of a structure per pound of payload or fuel consumption per mile. Subrahmaniam (1970) has made the study of product and quotient of order statistics from uniform distribution and exponential distribution. Malik and Trudel (1982) studied the cases when the order statistics are from Pareto, power and Weibull distributions. Aleem (2008) studied the quotient of two generalized order statistics from the Weibull distribution. Garg (2009) has studied the product and quotient of two generalized order statistics from Kumaraswami distribution. In this study, the distributions of the product and quotient of two generalized order statistics from the exponentiated Burr Type-XII distribution have been derived.

The concept of the Generalized Order Statistics (GOS) is introduced by Kamps (1995) as a unified approach to ordinary OS, record values and k-th record values, which can be outlined as:

The random variables $\mathrm{X}(1, \mathrm{n}, \mathrm{m}, \mathrm{k}), \ldots, \mathrm{X}(\mathrm{n}, \mathrm{n}, \mathrm{m}$, k) be GOS from an absolutely continuous CDF $F(x)$ and $\operatorname{PDF} \mathrm{f}(\mathrm{x})$, with noting that $(\mathrm{X}(0, \mathrm{n}, \mathrm{m}, \mathrm{k})=0, \geq 1$, $\left.\mathrm{m} \in \mathrm{R}^{\mathrm{n}-1}\right)$, then their joint PDF can be written in the form Equation 3:

$$
\begin{gathered}
f\left(x_{1}, x_{2}, \ldots, x_{n}\right)=k \prod_{i=1}^{n-1} \gamma_{i} f\left(x_{i}\right) \cdot\left[1-F\left(x_{i}\right)\right]^{m} \\
{\left[1-F\left(x_{n}\right)\right]^{k-1} f\left(x_{n}\right)}
\end{gathered}
$$

On the cone $\mathrm{F}^{-1}(0)<\mathrm{x}_{1}<\ldots<\mathrm{x}_{\mathrm{n}}<\mathrm{F}^{-1}(1)$ of $\mathrm{R}^{\mathrm{n}}$, where $\gamma_{\mathrm{i}}=\mathrm{k}+(\mathrm{n}-\mathrm{i})(\mathrm{m}+1)$ and $\gamma_{\mathrm{n}}=\mathrm{k}>0$.

If $\mathrm{m}=0$ and $\mathrm{k}=1$, then (1.3) is the joint PDF of the ordinary OS.

The joint PDF of $X(I, n, m, k)$ and $X(j, n, m, k), i<j$ is given by Equation 4:

$$
\begin{aligned}
f_{i, j}(x, y)= & \frac{c_{j-1}}{(i-1) !(j-i-1) !}(1-F(x))^{m} f(x) g_{m}^{i-1}(F(x)) \\
& \cdot\left[h_{m}(F(y))-h_{m}(F(x))\right]^{j-1-1}[1-F(y)]^{\gamma_{j}-1} f(y),
\end{aligned}
$$

For $\mathrm{x}<\mathrm{y}$.

Here $C_{j-i}=\prod_{i=1}^{j} \gamma_{i} \quad j=1,2, \ldots, n$ and $x \in[0,1], g_{m}(x)=$ $h_{m}(x)-h_{m}(0), h_{m}(x)=\left\{\begin{array}{cc}-\frac{1}{m+1}(1-x)^{m+1}, & m \neq-1 \\ -\log (1-x), & m=-1\end{array}\right.$

\subsection{The Mellin Transform}

Epstein (1948) was first to suggest a systematic approach to the study of products and quotients of independent random variables by using a Mellin transform technique.

The Mellin transform of $\mathrm{f}(\mathrm{x}, \mathrm{t})$ is defined as Equation 5:

$M_{s_{1}, s_{2}}(f(x, y))=\int_{0}^{¥} \int_{0}^{¥} x^{s_{1}-1} y^{s_{2}-1} f(x, y) d x d y$

where, $\mathrm{S}_{1}$ and $\mathrm{S}_{2}$ are complex variables. Under suitable conditions discussed by Fox (1957), it possesses the inverse as Equation 6:

$f(x, y)=\frac{1}{(2 \pi i)^{2}} \int_{h-i \infty \infty}^{h+i \infty} \int_{k-i \infty}^{k+i \infty} x^{-s_{1}} y^{-s_{2}} M_{s_{1}, s_{2}}(f(x, y)) d_{1} d s_{2}$

With the paths of integration being two lines parallel to the imaginary axis and to the right from the origin in the Argand plane.

We will study two cases:

- When $S_{1}=S_{2}=t$, we get:

$\mathrm{M}_{\mathrm{t}, \mathrm{t}}(\mathrm{g}(\mathrm{u}))=\mathrm{M}(\mathrm{t} \mid \mathrm{u})$, which corresponds to the Mellin transform for the PDF of the product $\mathrm{U}=\mathrm{XY}$. Thus Equation 7:

$g(u)=\frac{1}{2 \pi i} \int_{h-i \infty}^{h+i \infty} u^{-t} M(t \mid u) d t$

- When $\mathrm{S}_{1}=\mathrm{t}$ and $\mathrm{S}_{2}=-\mathrm{t}+2$, we get $\mathrm{M}_{\mathrm{t}, \mathrm{t}+2}(\mathrm{~h}(\mathrm{z}))=$ $\mathrm{M}(\mathrm{t} \mid \mathrm{z})$, which corresponds to the Mellin transform for the PDF of the quotient $\mathrm{Z}=\mathrm{X} / \mathrm{Y}$. Thus Equation 8 :

$h(z)=\frac{1}{2 \pi i} \int_{h-i \infty}^{h+i \infty} z^{-t} M(t \mid z) d t$ 


\subsection{The Fox H-Function}

The H-function is a generalization of the G-function and also it is defined, in the literature, as a Mellin-Barnes integral, Gradshteyn et al. (2007). The definitions of these functions will be defined as follows:

The G-function is defined as Equation 9:

$$
\begin{aligned}
& G_{p, q}^{m, n}\left[z \mid \begin{array}{l}
a_{1}, \ldots, a_{p} \\
b_{1}, \ldots, b_{q}
\end{array}\right]=\frac{1}{2 \pi i} \int_{h-i \infty}^{h+i \infty} z^{-t} g(t) d t \\
& g(t)=\frac{\prod_{j=1}^{m} \Gamma\left(b_{j}+t\right) \prod_{j=1}^{n} \Gamma\left(1-a_{j}-t\right)}{\prod_{j=m+1}^{q} \Gamma\left(1-b_{j}-t\right) \prod_{j=n+1}^{p} \Gamma\left(a_{j}+t\right)}
\end{aligned}
$$

The H-function is defined as Equation 10:

$$
\begin{aligned}
& \mathrm{H}_{\mathrm{p}, \mathrm{q}}^{\mathrm{m}, \mathrm{n}}\left[\mathrm{z}\left[\begin{array}{l}
\left(\mathrm{a}_{1}, \alpha_{1}\right), \ldots,\left(\mathrm{a}_{\mathrm{p}}, \alpha_{\mathrm{p}}\right) \\
\left.\left(\mathrm{b}_{1}, \beta_{1}\right), \ldots, \mathrm{b}_{\mathrm{q}}, \beta_{\mathrm{q}}\right)
\end{array}\right]=\frac{1}{2 \pi \mathrm{i}} \int_{\mathrm{h}-\mathrm{i} \neq}^{\mathrm{h}+\mathrm{i} \neq} \mathrm{z}^{-\mathrm{t}} \mathrm{h}(\mathrm{t}) \mathrm{dt}\right. \\
& \mathrm{h}(\mathrm{t})=\frac{\prod_{\mathrm{j}=1}^{\mathrm{m}} \Gamma\left(\mathrm{b}_{\mathrm{j}}+\beta_{\mathrm{j}} \mathrm{t}\right) \prod_{\mathrm{j}=1}^{\mathrm{n}} \Gamma\left(1-\mathrm{a}_{\mathrm{j}}-\alpha_{\mathrm{j}} \mathrm{t}\right)}{\prod_{\mathrm{j}=\mathrm{m}+1}^{\mathrm{q}} \Gamma\left(1-\mathrm{b}_{\mathrm{j}}-\beta_{\mathrm{j}} \mathrm{t}\right) \prod_{\mathrm{j}=\mathrm{n}+1}^{\mathrm{p}} \Gamma\left(\mathrm{a}_{\mathrm{j}}+\alpha_{\mathrm{j}} \mathrm{t}\right)}
\end{aligned}
$$

$\mathrm{m}, \mathrm{n}, \mathrm{p}$ and $\mathrm{q}$ are non-negative integers satisfying, $0 \leq \mathrm{n} \leq \mathrm{p}, 1 \leq \mathrm{m} \leq \mathrm{q} ; \alpha_{\mathrm{j}}(\mathrm{j}=1, \ldots, \mathrm{p})$ and $\beta_{\mathrm{j}}(\mathrm{j}=1, \ldots, \mathrm{q})$ are assumed to be positive quantities for standardization Purpose.

The inverse relation for the Fox function will be given as Equation 11:

$$
\begin{aligned}
& H_{p, q}^{m, n}\left[z \mid \begin{array}{c}
\left(a_{i}, \alpha_{i}\right), i=1, p \\
\left.\left(b_{j}, \beta_{j}\right), j=1, q\right)
\end{array}\right] \\
& =H_{p, q}^{m, n}\left[\frac{1}{z} \mid \begin{array}{c}
\left(1-b_{j}, \beta_{j}\right), j=1, q \\
\left.\left(1-a_{i}, \alpha_{i}\right), i=1, p\right)
\end{array}\right]
\end{aligned}
$$

\subsection{The Distribution of Generalized Order Statistics}

\subsubsection{The Distribution of Product of Two Generalized Order Statistics}

\section{Theorem 1}

Let $X(i, n, m, k)$ and $X(j, n, m, k)$ be the ith and $\mathrm{jth}$ generalized order statistics with $(i<j)$, based on a random sample of size $\mathrm{n}$ from the generalized exponentiated Burr Type-XII distribution, then the PDF of the product $U=X(i, n, m, k) \cdot X(j, n, m, k)$ is given by Equation 12:

$$
\begin{aligned}
& g(u)=\frac{(\theta \beta)^{2} c_{j-1}}{(i-1) !(j-i-1) !(m+1)^{j-2}} \\
& \sum_{\mathrm{r}, \mathrm{s}}^{*} \sum_{\mathrm{l}_{1}, 1_{2}}^{*} \frac{1}{\Gamma\left(\beta\left(\mathrm{l}_{2}+1\right)+1\right)} \mathrm{H}_{3,3}^{2,2}
\end{aligned}
$$

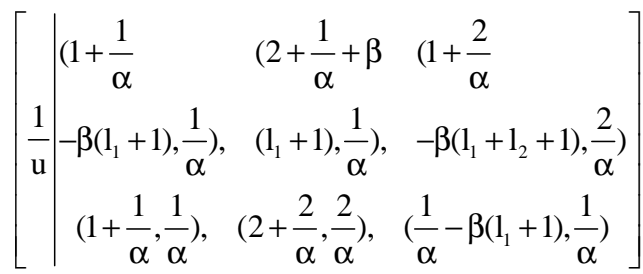

where, $\mathrm{O}<\mathrm{u} \leq \infty, \mathrm{H}[$.] is the Fox $\mathrm{H}$ - function defined by (10), $1 \leq \mathrm{i}<\mathrm{j} \leq \mathrm{n}$ and the symbol $\mathrm{c}_{\mathrm{j}-1}$ as defined in section (1).

\section{Proof}

For simplicity, let $\mathrm{X}$ and $\mathrm{Y}$ be the $\mathrm{i}$ th and $\mathrm{j}$ th GOS with $(i<j)$ from the exponentiated Burr Type-XII distribution (1). Thus, substituting (1) and (2) in (4), the joint PDF of these generalized order statistics is given by Equation 13:

$$
\begin{aligned}
& f(x, y)=\frac{c_{j-1}(\alpha \beta \theta)^{2}}{(i-1) !(j-i-1) !(m+1)^{j-2}} \\
& \sum_{\mathrm{r}=0}^{\mathrm{i}-1} \sum_{\mathrm{s}=0}^{\mathrm{j}-\mathrm{i}-1}(-1)^{\mathrm{r}+\mathrm{s}}\left(\begin{array}{c}
\mathrm{i}-1 \\
\mathrm{r}
\end{array}\right)\left(\begin{array}{c}
\mathrm{j}-\mathrm{i}-1 \\
\mathrm{~s}
\end{array}\right)
\end{aligned}
$$

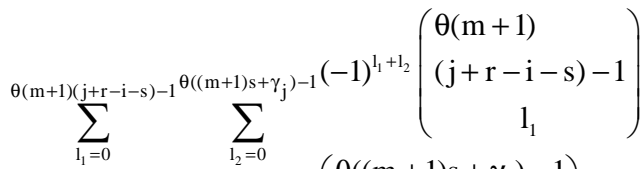

$$
\begin{aligned}
& \sum_{1_{1}=0} \quad\left(\begin{array}{c}
\theta\left((m+1) s+\gamma_{j}\right)-1 \\
1_{2}
\end{array}\right) \\
& x^{-\alpha-1} y^{-\alpha-1}\left(1+x^{-\alpha}\right)^{-\beta\left(l_{1}+1\right)-1}\left(1+y^{-\alpha}\right)^{-\beta\left(l_{2}+1\right)-1}
\end{aligned}
$$

The Mellin transform of (13) is given by:

$$
\begin{aligned}
& M_{s_{1}, s_{2}}(f(x, y))=K(\alpha \beta \theta)^{2} \sum_{r, s}^{*} \sum_{l_{1}, l_{2}}^{*} \\
& \int_{0}^{\infty} y^{s_{2}-\alpha-2}\left(1+y^{-\alpha}\right)^{-\beta\left(l_{2}+1\right)-1} I(y) d y
\end{aligned}
$$

Where: 


$$
\begin{aligned}
& K=\frac{c_{j-1}}{(i-1) !(j-i-1) !(m+1)^{j-2}}, \\
& \sum_{\mathrm{r}, \mathrm{s}}^{*}=\sum_{\mathrm{r}=0}^{\mathrm{i}-1} \sum_{\mathrm{s}=0}^{\mathrm{j}-\mathrm{j}-1}(-1)^{\mathrm{r}+\mathrm{s}}\left(\begin{array}{l}
\mathrm{i}-1 \\
\mathrm{r}
\end{array}\right)\left(\begin{array}{l}
\mathrm{j}-\mathrm{i}-1 \\
\mathrm{~s}
\end{array}\right), \\
& \sum_{1_{1}, l_{2}}^{*}=\sum_{1_{1}=0}^{\theta(m+1)(j+r-i-s)-1} \sum_{l_{2}=0}^{\theta\left((m+1) s+\gamma_{j}\right)-1}(-1)^{l_{1}+l_{2}}
\end{aligned}
$$

And:

$$
\mathrm{I}(\mathrm{y})=\int_{0}^{\mathrm{y}} \mathrm{x}^{\mathrm{s}_{1}-\alpha-2}\left(1+\mathrm{x}^{-\alpha}\right)^{-\beta\left(1_{1}+1\right)-1} \mathrm{dx} .
$$

Let $\mathrm{v}=\mathrm{x}^{-\alpha}$ and $\mathrm{w}=\mathrm{y}^{-\alpha}$, thus:

$$
\begin{gathered}
\mathrm{I}(\mathrm{y})=\frac{1}{\alpha} \int_{\mathrm{w}}^{\neq} \mathrm{t}^{\frac{\mathrm{s}_{1}-1}{\alpha}}(1+\mathrm{t})^{-\beta\left(\mathrm{l}_{1}+1\right)-1} \mathrm{dt} \\
=\frac{1}{\alpha} \frac{\mathrm{w}^{\left.\frac{\mathrm{s}_{1}-1}{\alpha}-\beta \mathrm{l}_{1}+1\right)}}{\left(\frac{\mathrm{s}_{1}-1}{\alpha}+\beta\left(1_{1}+1\right)\right)}{ }_{2} \mathrm{~F}_{1}\left(\beta\left(\mathrm{l}_{1}+1\right)+1,\right. \\
\left.\frac{\mathrm{s}_{1}-1}{\alpha}+\beta\left(1_{1}+1\right) ; \frac{\mathrm{s}_{1}-1}{\alpha}+\beta\left(l_{1}+1\right)+1 ;-\frac{1}{\mathrm{w}}\right)
\end{gathered}
$$

Gradshteyn et al. (2007), thus Equation 14:

$$
\begin{aligned}
& \mathrm{M}_{\mathrm{s}_{1}, \mathrm{~s}_{2}}(\mathrm{f}(\mathrm{x}, \mathrm{y}))=\mathrm{K}(\theta \beta)^{2}
\end{aligned}
$$

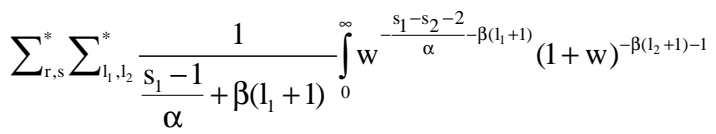

$$
\begin{aligned}
& { }_{2} \mathrm{~F}_{1}\left(\beta\left(1_{1}+1\right)+1, \frac{\mathrm{s}_{1}-1}{\alpha}+\beta\left(1_{1}+1\right)\right. \text {; } \\
& \left.\frac{\mathrm{s}_{1}-1}{\alpha}+\beta\left(1_{1}+1\right)+1 ;-\frac{1}{\mathrm{w}}\right) \mathrm{dw}
\end{aligned}
$$

Let $\mathrm{v}=-\frac{1}{\mathrm{w}}$ and using the integral in Gradshteyn et al. (2007), the Mellin transform (14) can be written as Equation 15:

$$
\begin{aligned}
& \mathrm{M}_{\mathrm{s}_{1}, \mathrm{~s}_{2}}(\mathrm{f}(\mathrm{x}, \mathrm{y}))=\mathrm{K}(\theta \beta)^{2} \sum_{\mathrm{r}, \mathrm{s}}^{*} \sum_{\mathrm{l}_{1}, 1_{2}}^{*} \frac{1}{\frac{\mathrm{s}_{1}-1}{\alpha}+\beta\left(1_{1}+1\right)} \\
& \Gamma\left(\frac{\mathrm{s}_{1}+\mathrm{s}_{2}-2}{\alpha}+\beta\left(\mathrm{l}_{1}+1_{2}+2\right)\right) \\
& \frac{\Gamma\left(-\frac{\mathrm{s}_{1}+\mathrm{s}_{2}-2}{\alpha}+2\right) \Gamma\left(-\frac{\mathrm{s}_{2}-1}{\alpha}+1\right)}{\Gamma\left(\beta\left(1_{2}+1\right)+1\right) \Gamma\left(\beta\left(1_{1}+1\right)-\frac{\mathrm{s}_{2}-1}{\alpha}+2\right)}
\end{aligned}
$$

By setting $\mathrm{s}_{1}=\mathrm{t}$ and $\mathrm{s}_{2}=\mathrm{t}$ in (15), we get $\left.\mathrm{M}_{\mathrm{t}, \mathrm{t}} \mathrm{g}(\mathrm{u})\right)$ $=\mathrm{M}(\mathrm{t} \mid \mathrm{u})$, which corresponds to the Mellin transform for the $\mathrm{PDF}$ of the product $\mathrm{U}=\mathrm{XY}$. Thus Equation 16:

$$
\begin{aligned}
& \mathrm{M}_{\mathrm{t}, \mathrm{t}}(\mathrm{g}(\mathrm{u}))=\mathrm{M}(\mathrm{t} \mid \mathrm{u})=\mathrm{K}(\theta \beta)^{2} \sum_{\mathrm{r}, \mathrm{s}}^{*} \sum_{\mathrm{l}_{1}, \mathrm{l}_{2}}^{*} \frac{1}{\mathrm{t}-1} \frac{1}{\alpha}+\beta\left(\mathrm{l}_{1}+1\right) \\
& \frac{\Gamma\left(\frac{2 \mathrm{t}-2}{\alpha}+\beta\left(\mathrm{l}_{1}+\mathrm{l}_{2}+2\right)\right) \Gamma\left(-\frac{2 \mathrm{t}-2}{\alpha}+2\right) \Gamma\left(-\frac{\mathrm{t}-1}{\alpha}+1\right)}{\Gamma\left(\beta\left(1_{2}+1\right)+1\right) \Gamma\left(\beta\left(1_{1}+1\right)-\frac{\mathrm{t}-1}{\alpha}+2\right)} \\
& =\mathrm{K}(\theta \beta)^{2} \sum_{\mathrm{r}, \mathrm{s}}^{*} \sum_{\mathrm{l}_{1} \mathrm{l}_{2}}^{*} \\
& \frac{\Gamma\left(\beta\left(1_{1}+1\right)+\frac{\mathrm{t}-1}{\alpha}\right) \Gamma\left(\frac{2 \mathrm{t}-2}{\alpha}+\beta\left(\mathrm{l}_{1}+1_{2}+2\right)\right)}{\Gamma\left(-\frac{2 \mathrm{t}-2}{\alpha}+2\right) \Gamma\left(-\frac{\mathrm{t}-1}{\alpha}+1\right)} \\
& \Gamma\left(\beta\left(1_{1}+1\right)+\frac{\mathrm{t}-1}{\alpha}+1\right) \\
& \Gamma\left(\beta\left(1_{2}+1\right)+1\right) \Gamma\left(\beta\left(1_{1}+1\right)-\frac{\mathrm{t}-1}{\alpha}+2\right)
\end{aligned}
$$

Thus, using (7) with (16), we get the PDF of the product $\mathrm{U}$ as:

$$
\begin{aligned}
& \mathrm{g}(\mathrm{u})=\mathrm{K}(\theta \beta)^{2} \sum_{\mathrm{r}, \mathrm{s}}^{*} \sum_{\mathrm{l}_{1}, \mathrm{l}_{2}}^{*} \frac{1}{\Gamma\left(\beta\left(\mathrm{l}_{2}+1\right)+1\right)} \\
& \Gamma\left(\beta\left(1_{1}+1\right)+\frac{\mathrm{t}-1}{\alpha}\right) \Gamma\left(\frac{2 \mathrm{t}-2}{\alpha}+\beta\left(1_{1}+1_{2}+2\right)\right) \\
& \frac{1}{2 \pi \mathrm{i}} \int_{\mathrm{h}-\mathrm{i} \neq}^{\mathrm{h}+\mathrm{i}} \mathrm{u}^{-\mathrm{t}} \frac{\Gamma\left(-\frac{2 \mathrm{t}-2}{\alpha}+2\right) \Gamma\left(-\frac{\mathrm{t}-1}{\alpha}+1\right)}{\Gamma\left(\beta\left(1_{1}+1\right)+\frac{\mathrm{t}-1}{\alpha}+1\right) \Gamma\left(\beta\left(1_{1}+1\right)-\frac{\mathrm{t}-1}{\alpha}+2\right)} \mathrm{dt} \\
& \mathrm{g}(\mathrm{u})=\mathrm{K}(\theta \beta)^{2} \sum_{\mathrm{r}, \mathrm{s}}^{*} \sum_{\mathrm{l}_{1}, \mathrm{l}_{2}}^{*} \frac{1}{\Gamma\left(\beta\left(\mathrm{l}_{2}+1\right)+1\right)} \\
& \mathrm{H}_{3,3}^{2,2}\left[\begin{array}{l}
\left(-\frac{1}{\alpha}, \frac{1}{\alpha}\right), \quad\left(-1-\frac{2}{\alpha}, \frac{2}{\alpha}\right), \quad\left(1-\frac{1}{\alpha}+\beta\left(1_{1}+1\right), \frac{1}{\alpha}\right) \\
\mathrm{u} \\
\left(\beta\left(1_{1}+1\right)-\frac{1}{\alpha}, \frac{1}{\alpha}\right), \\
\left(-1-\frac{1}{\alpha}-\beta\left(1_{1}+1\right), \frac{1}{\alpha}\right),\left(-\frac{2}{\alpha}+\beta\left(1_{1}+l_{2}+2\right), \frac{2}{\alpha}\right)
\end{array}\right]
\end{aligned}
$$

Using the inverse relation (11) we get the PDF of the product (12).

\subsection{The Distribution of Quotient of Two Generalized Order Statistics}

\section{Theorem 2:}

Let $X(i, n, m, k)$ and $X(j, n, m, k)$ be the ith and $j$ th generalized order statistics with $(i<j)$, based on a 
random sample of size $\mathrm{n}$ from the generalized Burr Type-XII distribution, then the PDF of the quotient $\mathrm{Z}=$ $\mathrm{X}(\mathrm{i}, \mathrm{n}, \mathrm{m}, \mathrm{k}) / \mathrm{X}(\mathrm{j}, \mathrm{n}, \mathrm{m}, \mathrm{k})$ is given by Equation 17:

$$
\begin{aligned}
& h(z)=\frac{(\theta \beta)^{2} c_{j-1}}{(i-1) !(j-i-1) !(m+1)^{j-2}} \sum_{r, s}^{*} \sum_{l_{1}, l_{2}}^{*} \frac{\Gamma\left(\beta\left(l_{1}+l_{2}+2\right)\right)}{\Gamma\left(\beta\left(l_{2}+1\right)+1\right)}
\end{aligned}
$$

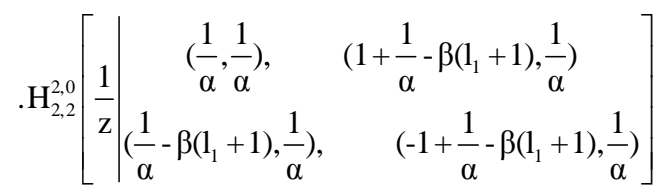

where, $\mathrm{O}<\mathrm{Z} \leq \infty, 1 \leq \mathrm{i}<\mathrm{j} \leq \mathrm{n}$ and the symbol $\mathrm{c}_{\mathrm{j}-1}$ as defined in section 1 .

\section{Proof}

Using the Mellin transform (15) by setting $\mathrm{s}_{1}=\mathrm{t}$ and $\mathrm{s}_{2}=\mathrm{t}+2$, we get $\mathrm{M}_{\mathrm{t}, \mathrm{t}+2}(\mathrm{~h}(\mathrm{z}))=\mathrm{M}(\mathrm{t} \mid \mathrm{z})$, which corresponds to the Mellin transform for the PDF of the quotient $\mathrm{Z}=\mathrm{X} / \mathrm{Y}$. Thus Equation 18 :

$$
\begin{aligned}
& \mathrm{M}_{\mathrm{t}, \mathrm{t}+2}(\mathrm{~h}(\mathrm{z}))=\mathrm{M}(\mathrm{t} \mid \mathrm{z}) \\
& =\mathrm{K}(\theta \beta)^{2} \sum_{\mathrm{r}, \mathrm{s}}^{*} \sum_{\mathrm{l}_{1}, \mathrm{l}_{2}}^{*} \frac{1}{\frac{\mathrm{t}-1}{\alpha}+\beta\left(1_{1}+1\right)} \\
& \frac{\Gamma\left(\beta\left(1_{1}+1_{2}+2\right)\right) \Gamma(2) \Gamma\left(\frac{\mathrm{t}-1}{\alpha}+1\right)}{\Gamma\left(\beta\left(1_{2}+1\right)+1\right) \Gamma\left(\beta\left(1_{1}+1\right)+\frac{\mathrm{t}-1}{\alpha}+2\right)}
\end{aligned}
$$

Thus, from (8) with (19) we get the PDF of the quotient as:

$$
\begin{aligned}
& \mathrm{h}(\mathrm{z})=\mathrm{K}(\alpha \theta)^{2} \sum_{\mathrm{r}, \mathrm{s}}^{*} \sum_{\mathrm{l}_{1}, \mathrm{l}_{2}}^{*} \frac{\Gamma\left(\beta\left(\mathrm{l}_{1}+\mathrm{l}_{2}+2\right)\right)}{\Gamma\left(\beta\left(\mathrm{l}_{2}+1\right)+1\right)} \\
& \frac{1}{2 \pi \mathrm{i}} \int_{\mathrm{h}-\mathrm{i} \infty}^{\mathrm{h}+\mathrm{i} \infty} \mathrm{z}^{-\mathrm{t}} \frac{\Gamma\left(\frac{\mathrm{t}-1}{\alpha}+1\right) \Gamma\left(\beta\left(\mathrm{l}_{1}+1\right)+\frac{\mathrm{t}-1}{\alpha}\right)}{\Gamma\left(\beta\left(\mathrm{l}_{1}+1\right)+\frac{\mathrm{t}-1}{\alpha}+1\right) \Gamma\left(\beta\left(\mathrm{l}_{1}+1\right)+\frac{\mathrm{t}-1}{\alpha}+2\right)} \mathrm{dt} \\
& \mathrm{h}(\mathrm{z})=\mathrm{K}(\theta \beta)^{2} \sum_{\mathrm{r}, \mathrm{s}}^{*} \sum_{\mathrm{l}_{1}, \mathrm{l}_{2}}^{*} \frac{\Gamma\left(\beta\left(\mathrm{l}_{1}+\mathrm{l}_{2}+2\right)\right)}{\Gamma\left(\beta\left(\mathrm{l}_{2}+1\right)+1\right)} \\
& . \mathrm{H}_{2,2}^{2,0}\left[\begin{array}{c}
\mathrm{z}\left[\begin{array}{c}
\left(1-\frac{1}{\alpha}+\beta\left(\mathrm{l}_{1}+1\right), \frac{1}{\alpha}\right), \\
\left(1-\frac{1}{\alpha}, \frac{1}{\alpha}\right),
\end{array} \quad\left(\beta\left(\mathrm{l}_{1}+1\right)-\frac{1}{\alpha}, \frac{1}{\alpha}\right)\right.
\end{array}\right]
\end{aligned}
$$

Using the inverse relation (11) we get the PDF of the quotient (18).

\section{Corollary 1}

Let $\mathrm{I}=1$ and $\mathrm{j}=\mathrm{n}$ in (12) and (18) we get the density functions of the product and quotient of the extreme generalized order statistics from the exponentiated Burr Type XII distribution respectively as:

$$
\begin{aligned}
& \mathrm{g}(\mathrm{u})=\frac{(\theta \beta)^{2} \mathrm{c}_{\mathrm{n}-1}}{(\mathrm{n}-2) !(\mathrm{m}+1)^{\mathrm{n}-2}} \sum_{\mathrm{r}, \mathrm{s}}^{* *} \sum_{\mathrm{l}_{1}, \mathrm{l}_{2}}^{* *} \frac{1}{\Gamma\left(\beta\left(\mathrm{l}_{2}+1\right)+1\right)} \\
& \mathrm{H}_{3,3}^{2,2}\left[\frac{1}{\mathrm{u}}\left[\begin{array}{c}
\left(1+\frac{1}{\alpha}-\beta\left(1_{1}+1\right), \frac{1}{\alpha}\right), \quad\left(2+\frac{1}{\alpha}+\beta\left(1_{1}+1\right), \frac{1}{\alpha}\right), \\
\left(1+\frac{2}{\alpha}\left(1_{1}+1_{2}+1\right), \frac{2}{\alpha}\right) \\
\left(1+\frac{1}{\alpha}, \frac{1}{\alpha}\right), \quad\left(2+\frac{2}{\alpha}, \frac{2}{\alpha}\right), \quad\left(\frac{1}{\alpha}-\beta\left(1_{1}+1\right), \frac{1}{\alpha}\right)
\end{array}\right]\right. \\
& \mathrm{h}(\mathrm{z})=\frac{(\theta \beta)^{2} \mathrm{c}_{\mathrm{n}-1}}{(\mathrm{n}-2) !(\mathrm{m}+1)^{\mathrm{n}-2}} \sum_{\mathrm{r}, \mathrm{s}}^{* *} \sum_{\mathrm{l}_{1}, \mathrm{l}_{2}}^{* *} \frac{\Gamma\left(\beta\left(\mathrm{l}_{1}+\mathrm{l}_{2}+2\right)\right)}{\Gamma\left(\beta\left(\mathrm{l}_{2}+1\right)+1\right)} \\
& . H_{2,2}^{2,0}\left[\frac{1}{\mathrm{z}}\left[\begin{array}{cc}
\left(\frac{1}{\alpha}, \frac{1}{\alpha}\right), & \left(1+\frac{1}{\alpha}-\beta\left(l_{1}+1\right), \frac{1}{\alpha}\right) \\
\left(\frac{1}{\alpha}-\beta\left(l_{1}+1\right), \frac{1}{\alpha}\right), & \left(-1+\frac{1}{\alpha}-\beta\left(l_{1}+1\right), \frac{1}{\alpha}\right)
\end{array}\right]\right.
\end{aligned}
$$

Where:

$$
\begin{gathered}
\sum_{\mathrm{s}}^{* *}=\sum_{\mathrm{s}=0}^{\mathrm{n}-2}(-1)^{\mathrm{s}}\left(\begin{array}{c}
\mathrm{n}-2 \\
\mathrm{~s}
\end{array}\right) \\
\sum_{\mathrm{l}_{1}, \mathrm{l}_{2}}^{* *}=\sum_{1_{1}=0}^{\theta(\mathrm{m}+1)(\mathrm{n}-\mathrm{s}-1)-1} \sum_{\mathrm{l}_{2}=0}^{\theta((\mathrm{m}+1) \mathrm{s}+\mathrm{k})-1}(-1)^{\mathrm{l}_{1}+\mathrm{l}_{2}}\left(\begin{array}{l}
\theta(\mathrm{m}+1)(\mathrm{n}-\mathrm{s}-1)-1 \\
\mathrm{l}_{1}
\end{array}\right)\left(\begin{array}{l}
\theta((\mathrm{m}+1) \mathrm{s}+\mathrm{k})-1 \\
\mathrm{l}_{2}
\end{array}\right)
\end{gathered}
$$

Setting $\theta=1$, we get the density functions of the product and quotient of the extreme generalized order statistics from the standard Burr Type XII distribution.

\section{Corollary 2}

Let $\mathrm{j}=\mathrm{i}+1$ in (4.1) and (5.1) we get the density functions of the product and quotient of the consecutive generalized order statistics $(i<j)$ from the exponentiated Burr Type XII distribution respectively as:

$$
\begin{aligned}
& \mathrm{g}(\mathrm{u})=\frac{(\theta \beta)^{2} \mathrm{c}_{\mathrm{i}}}{(\mathrm{i}-1) !(\mathrm{m}+1)^{\mathrm{i}-1}} \sum_{\mathrm{r}}^{* * *} \sum_{\mathrm{l}_{1}, \mathrm{l}_{2}}^{* * * *} \frac{1}{\Gamma\left(\beta\left(\mathrm{l}_{2}+1\right)+1\right)}
\end{aligned}
$$

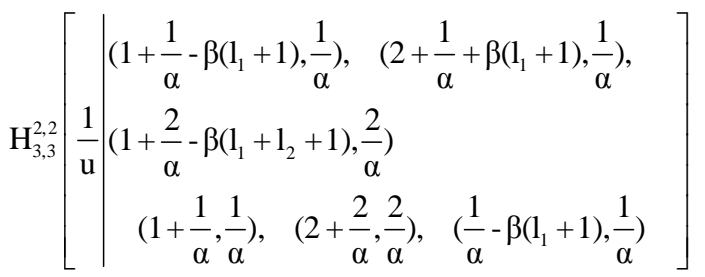




$$
\begin{aligned}
& \mathrm{h}(\mathrm{z})=\frac{(\theta \beta)^{2} \mathrm{c}_{\mathrm{i}}}{(\mathrm{i}-1) !(\mathrm{m}+1)^{\mathrm{i}-1}} \sum_{\mathrm{r}}^{* *} \sum_{\mathrm{l}_{1}, \mathrm{l}_{2}}^{* * * *} \frac{\Gamma\left(\beta\left(\mathrm{l}_{1}+\mathrm{l}_{2}+2\right)\right)}{\Gamma\left(\beta\left(\mathrm{l}_{2}+1\right)+1\right)} \\
& . \mathrm{H}_{2,2}^{2,0}\left[\frac{1}{\mathrm{z}} \mid \begin{array}{cc}
\left(\frac{1}{\alpha}, \frac{1}{\alpha}\right), & \left(1+\frac{1}{\alpha}-\beta\left(l_{1}+1\right), \frac{1}{\alpha}\right) \\
\left(\frac{1}{\alpha}-\beta\left(l_{1}+1\right), \frac{1}{\alpha}\right), & \left(-1+\frac{1}{\alpha}-\beta\left(l_{1}+1\right), \frac{1}{\alpha}\right)
\end{array}\right]
\end{aligned}
$$

Where:

$$
\begin{gathered}
\sum_{\mathrm{r}}^{* * *}=\sum_{\mathrm{r}=0}^{\mathrm{i}-1}(-1)^{\mathrm{r}}\left(\begin{array}{l}
\mathrm{i}-1 \\
\mathrm{r}
\end{array}\right) \\
\sum_{\mathrm{l}_{1}, \mathrm{l}_{2}}^{* * * *}=\sum_{\mathrm{l}_{1}=0}^{\theta(\mathrm{m}+1)(\mathrm{r}+1)-1} \sum_{\mathrm{l}_{2}=0}^{\theta \gamma_{\mathrm{i}+1}-1}(-1)^{\mathrm{l}_{1}+\mathrm{l}_{2}}\left(\begin{array}{l}
\theta(\mathrm{m}+1)(\mathrm{r}+1)-1 \\
\mathrm{l}_{1}
\end{array}\right)\left(\begin{array}{l}
\theta \gamma_{\mathrm{l}_{2}}-1 \\
\mathrm{l}_{1}-1
\end{array}\right)
\end{gathered}
$$

Setting $\theta=1$, we get the density functions of the product and quotient for consecutive generalized order statistics from the standard Burr Type XII distribution.

\section{Corollary 3}

When $\theta=1,(12)$ and (18) reduce to the density functions of the product and quotient of the $i$ th and $j$ th generalized order statistics from the Burr Type XII distribution respectively as:

$$
\begin{aligned}
& g(u)=\frac{\beta^{2} c_{j-1}}{(i-1) !(j-i-1) !(m+1)^{j-2}} \sum_{r, s}^{*} \sum_{l_{1}, l_{2}}^{\prime} \frac{1}{\Gamma\left(\beta\left(l_{2}+1\right)+1\right)} \\
& \mathrm{H}_{3,3}^{2,2}\left[\frac{1}{\mathrm{u}}\left[\begin{array}{c}
\left(1+\frac{1}{\alpha}-\beta\left(1_{1}+1\right), \frac{1}{\alpha}\right), \quad\left(2+\frac{1}{\alpha}+\beta\left(1_{1}+1\right), \frac{1}{\alpha}\right), \\
\left.\alpha-\beta\left(1_{1}+1_{2}+1\right), \frac{2}{\alpha}\right) \\
\left(1+\frac{1}{\alpha}, \frac{1}{\alpha}\right), \quad\left(2+\frac{2}{\alpha}, \frac{2}{\alpha}\right), \quad\left(\frac{1}{\alpha}-\beta\left(l_{1}+1\right), \frac{1}{\alpha}\right)
\end{array}\right]\right. \\
& \mathrm{h}(\mathrm{z})=\frac{\beta^{2} \mathrm{c}_{\mathrm{j}-1}}{(\mathrm{i}-1) !(\mathrm{j}-\mathrm{i}-1) !(\mathrm{m}+1)^{\mathrm{j}-2}} \sum_{\mathrm{r}, \mathrm{s}}^{*} \sum_{\mathrm{l}_{1}, \mathrm{l}_{2}}^{\prime} \frac{\Gamma\left(\beta\left(\mathrm{l}_{1}+\mathrm{l}_{2}+2\right)\right)}{\Gamma\left(\beta\left(\mathrm{l}_{2}+1\right)+1\right)} \\
& \mathrm{H}_{2,2}^{2,0}\left[\frac{1}{\mathrm{z}}\left[\begin{array}{cc}
\left(\frac{1}{\alpha}, \frac{1}{\alpha}\right), & \left(1+\frac{1}{\alpha}-\beta\left(l_{1}+1\right), \frac{1}{\alpha}\right) \\
\left(\frac{1}{\alpha}-\beta\left(l_{1}+1\right), \frac{1}{\alpha}\right), & \left(-1+\frac{1}{\alpha}-\beta\left(l_{1}+1\right), \frac{1}{\alpha}\right)
\end{array}\right]\right.
\end{aligned}
$$

Where:

$$
\sum_{1_{1}, 1_{2}}^{\prime}=\sum_{1_{1}=0}^{(m+1)(r+1)-1} \sum_{1_{2}=0}^{\gamma_{i+1}-1}(-1)^{l_{1}+l_{2}}\left(\begin{array}{l}
(m+1)(r+1)-1 \\
1_{1}
\end{array}\right)\left(\begin{array}{l}
\gamma_{1}+1 \\
l_{2}-1
\end{array}\right)
$$

\section{Corollary 4}

When $\theta=1$ and $\mathrm{m}=0$ and $\mathrm{k}=1$ (12) and (18) reduce to the density functions of the product and the quotient of the $i$ th and $j$ th order statistics from the ordinary Burr Type XII distribution respectively as:

$$
\begin{aligned}
& g(u)=\frac{\beta^{2} n !}{(i-1) !(j-i-1) !(n-j) !} \sum_{r, s}^{*} \sum_{1_{1}, l_{2}}^{\prime \prime} \frac{1}{\Gamma\left(\beta\left(l_{2}+1\right)+1\right)} \\
& \mathrm{H}_{3,3}^{2,2}\left[\frac{1}{\mathrm{u}}\left[\begin{array}{c}
\left(1+\frac{1}{\alpha}-\beta\left(l_{1}+1\right), \frac{1}{\alpha}\right), \quad\left(2+\frac{1}{\alpha}+\beta\left(l_{1}+1\right), \frac{1}{\alpha}\right), \\
\left(1+\frac{2}{\alpha}-\beta\left(l_{1}+1_{2}+1\right), \frac{2}{\alpha}\right) \\
\left(1+\frac{1}{\alpha}, \frac{1}{\alpha}\right), \quad\left(2+\frac{2}{\alpha}, \frac{2}{\alpha}\right), \quad\left(\frac{1}{\alpha}-\beta\left(1_{1}+1\right), \frac{1}{\alpha}\right)
\end{array}\right]\right. \\
& \mathrm{h}(\mathrm{z})=\frac{\beta^{2} \mathrm{n} !}{(\mathrm{i}-1) !(\mathrm{j}-\mathrm{i}-1) !(\mathrm{n}-\mathrm{j}) !} \sum_{\mathrm{r}, \mathrm{s}}^{*} \sum_{\mathrm{l}_{1}, \mathrm{l}_{2}}^{\prime \prime} \frac{\Gamma\left(\beta\left(\mathrm{l}_{1}+\mathrm{l}_{2}+2\right)\right)}{\Gamma\left(\beta\left(\mathrm{l}_{2}+1\right)+1\right)} \\
& \cdot \mathrm{H}_{2,2}^{2,0}\left[\frac{1}{\mathrm{z}} \mid \begin{array}{cc}
\left(\frac{1}{\alpha}, \frac{1}{\alpha}\right), & \left(1+\frac{1}{\alpha}-\beta\left(l_{1}+1\right), \frac{1}{\alpha}\right) \\
\left(\frac{1}{\alpha}-\beta\left(l_{1}+1\right), \frac{1}{\alpha}\right), & \left(-1+\frac{1}{\alpha}-\beta\left(l_{1}+1\right), \frac{1}{\alpha}\right)
\end{array}\right]
\end{aligned}
$$

Where:

$$
\sum_{1_{1}, 1_{2}}^{\prime \prime}=\sum_{1_{1}=0}^{(j+r-i-s-1)} \sum_{1_{2}=0}^{n-j+s}(-1)^{1_{1}+l_{2}}\left(\left(_{1_{1}}^{j+r-i-s-1}\right)\left(n_{l_{2}}^{n-j+s}\right)\right.
$$

$\operatorname{Garg}$ (2009) when $\alpha=-a$ and $\beta=-b$.

\section{CONCLUSION}

In this study, we have proposed a new family of distributions called exponentiated Burr type-XII distribution. We get the probability density functions for the product and Quotient of two generalized statistics from this distribution. We have derived some interesting special cases such as the product and quotient of extreme generalized order statistics and the product and quotient of consecutive generalized order statistics.

These functions are useful in the investment in a number of different overseas markets and the problem of selection and ranking rules.

\section{ACKNOWLEDGMENT}

The researchers would like to thank the editor and the two referees for their helpful comments and suggestions.

\section{REFERENCES}

Aleem, M., 2008. On probability density function of the quotient of generalized order statistics from the weibull distribution. J. Stat., 15: 17-25. 
Burr, I.W., 1942. Cumulative frequency functions. Ann. Math., 13: 215-232.

Burr, I.W., 1976. The effect of non-normality on constants for X and R charts. Indus. Q. Control.

Cook, D.R. and E.S. Johnson, 1986. Generalized burrpareto-logestic distributions with applications to a uranium exploration data set. Technometrics, 28: 123-131. DOI: 10.1080/00401706.1986.10488113

Epstein, B., 1948. Some applications of the Mellin transform in statistics. Ann. Math. Stat., 19: 370379. DOI: $10.1214 /$ aoms/1177730201

Evans, L.G. and A.S. Ragab, 1983. Bayesian inferences given a type-II censored sample from a BURR distribution. Commun. Stat. Theory Meth., A12: 1569-1580.

Evans, R.A. and G. Simons, 1975. Research on statistical procedures in reliability engineering. ARL, TR.

Fox, C., 1957. Some applications of Mellin transforms to the theory of Bivariate statistical distributions. Proc. Cambridge Philosophical Soc., 53: 620-628. DOI: 10.1017/S0305004100032679

Garg, M., 2009. On generalized order statistics from kumaraswamy distribution. Tamsui Oxford J. Math. Sci., 25: 153-166.

Gradshteyn, I.S., I.M. Ryzhik, A. Jeffrey and D. Zwillinger, 2007. Table of Integrals, Series and Products. 7th Edn., Academic Press, Amsterdam, Boston, ISBN-10: 0123736374, pp: 1171.

Gulati, L., 1970. On the distributions of the products of order statistics. Annals Math. Stat., 41: 1156-1167.

Gupta, R.D. and D. Kundu, 1999. Generalized exponential distribution. Australia and New Zealand J. Stat., 41: 173-188. DOI: 10.1111/1467842X.00072

Gupta, R.D.D. and D. Kundu, 2001. Exponentiated exponential family: An alternative to gamma and Weibull. Biometr. J., 43: 117-130. DOI: 10.1002/1521-4036(200102)43:1<117::AID$\mathrm{BIMJ} 117>3.0 . \mathrm{CO} ; 2-\mathrm{R}$

Kamps, U., 1995. A Concept of generalized order statistics. J. Stat. Plann. Inference, 48: 1-23. DOI: 10.1016/0378-3758(94)00147-N

Lee, W.C., J.W. Wu and C.W. Hong, 2009. Assessing the lifetime performance index of products from progressively type II right censored data using Burr XII model. Math. Comput. Simul., 79: 2167-2179. DOI: 10.1016/j.matcom.2008.12.001

Malik, H.J. and R. Trudel, 1982. Probability density function of quotient of order statistics from the pareto, power and weibull distributions. Commun. Stat. Theor., 11: 801-814. DOI: $10.1080 / 03610928208828272$
Malik, H.J., 1970. The distribution of the products of two non central Beta variates. Naval Res. Logistics Q., 17: 327-330. DOI: 10.1002/nav.3800170309

McDonald, J.B. and D.O. Richards, 1987. Model selection, some generalized distributions. Commun. Stat. Theory Mthods, 16: 1049-1047. DOI: 10.1080/03610928708829422

Mielke, P.W. Jr. and E.S. Johnson, 1974. Some generalized beta distributions of the second kind having desirable applications features in hydrology and meterology. Water Resources Res., 10: 223-226. DOI: 10.1029/WR010i002p00223

Mudholkar, G.S. and D.K. Srivastava, 1993. Exponentiated Weibull family for analyzing bathtub failure rate data. IEEE Trans. Reliab., 42: 299-302. DOI: $10.1109 / 24.229504$

Nadarajah, S. and A. Gupta, 2005a. On the product and ratio of Bessel random variables. Int. J. Math. Math. Sci., 18: 2977-2989. DOI: 10.1155/IJMMS.2005.2977

Nadarajah, S. and A.K. Gupta, 2005b. On the moments of the exponentiated Weibull distribution. Commun. Stat.: Theory Methods, 34: 253-256. DOI: 10.1080/03610920509342418

Nadarajah, S. and S. Kotz, 2007. On the product and ratio of $\mathrm{t}$ and Bessel random variables. Bull. Instit. Math. Acad. Sinica, 2: 55-66.

Nadarajah, S., 2005. On the product and ratio of Laplce and Bessel random variables. J. Applied Math., 4: 393-402. DOI: 10.1155/JAM.2005.393

Nagar, D.K. and D.B. Valencia, 2011. Product and quotient of independent gauss hypergeometric variables. Ingenieray Ciencia, 7: 29-48.

Rodiguez, R.N., 1977. Aguide to the Burr type-XII distributions. Biometrika, 64: 129-134. DOI: 10.1093/biomet/64.1.129

Rodriguez, R.N. and B.Y. Taniguchi, 1980. New statistical model for predicting customer octane satisfaction using trained-rater observations. Trans. Soc. Automutive Eng.

Soliman, A.S., 2002. Reliability estimations in a generalized life-model with application to the BurrXII. IEEE Trans. Reliab., 51: 337-343. DOI: 10.1109/TR.2002.801855

Subrahmaniam, K., 1970. On some applications of Mellin transforms to statistics: Dependent random variables. SIAM J. Applied Math., 19: 658-662. DOI: $10.1137 / 0119064$

Tang, J. and A.K. Gupta, 1984. On the distribution of the product of independent beta random variables. Stat. Probab. Lett., 2: 165-168. DOI: 10.1016/01677152(84)90008-7 
Wallgren, C.M., 1980. The distribution of the product of two correlated t variates. J. Am. Stat. Assoc., 75: 996-1000. DOI: 10.1080/01621459.1980.10477585

Wang, L. and Y. Shi, 2010. Empirical inference for the burr model based on records. Applied Math. Sci., 4: 1663-1670.

Wingo, D.R., 1993. Maximum likelihood estimation of the Burr XII distribution parameters under type-II censoring. Microelectorn Reliab., 23: 1251-1257. DOI: 10.1016/0026-2714(93)90126-J
Wu, J.W. and H.Y. Yu, 2005. Statistical Inference about shape parameter of the new two-parameter of the Burr XII distribution under the failure-censored sampling Plan. Applied Math. Comput., 163: 443482. DOI: 10.1016/j.amc.2004.02.019

Xiuchun, L., S. Yimin, W. Jieqiong and C. Jian, 2007. Empirical Bayes estimators of reliability performances using Linex loss under progressively Type-II censored samples. Math. Comput. Simul., 73: 320-326. DOI: 10.1016/j.matcom.2006.05.002 